by an additional $0.06(95 \% \mathrm{CI}, 0.04$ to 0.08$) \mathrm{kg} / \mathrm{m}^{2}, 0.03$ (95\% CI, 0.01 to 0.05$) \mathrm{kg} / \mathrm{m}^{2}, 0.20$ (95\% CI, 0.11 to 0.28 ) $\mathrm{kg}$ and 0.17 (95\% CI, 0.11 to 0.22$) \mathrm{cm}$ per year among those in the highest quintile of ultra-processed food consumption compared with their lowest quintile counterpart. Evidence of dose-response relationships were consistently observed with those in the two highest quintiles of ultra-processed food consumption showing significantly more rapid progression of body mass index, weight, and waist circumference.

Conclusion These findings provide important and novel evidence that higher consumption of ultra-processed foods is associated with more rapid progression of adiposity from childhood to early adulthood. Radical and effective public health actions that reduce children's exposure and consumption of ultra-processed foods and encourage greater consumption of minimally processed foods are urgently needed to counteract the growing burden of obesity in England and globally.

\section{OP38 FRUIT AND VEGETABLES FISCAL POLICIES FOR REDUCING CARDIOVASCULAR MORTALITY AND RELATED INEQUALITIES: A MODELLING STUDY IN A LARGE SOUTHERN EUROPEAN URBAN POPULATION}

${ }^{1}$ Carlos Fernández-Escobar*, ${ }^{2} J u l i a$ Díez, ${ }^{3}$ Alba Martínez-García, ${ }^{4}$ Beatriz González LópezValcárcel, ${ }^{5}$ Simon Capewell, ${ }^{2}$ Manuel Franco, ${ }^{5}$ Martin O'Flaherty. ${ }^{1}$ Escuela Nacional de Sanidad, Instituto de Salud Carlos III, Madrid, Spain; ${ }^{2}$ Departamento de Cirugía, Ciencias Médicas y Sociales, Universidad de Alcalá, Alcalá de Henares, Spain; ${ }^{3}$ Departamento de Enfermería Comunitaria, Medicina Preventiva y Salud Pública, Universidad de Alicante, Alicante, Spain; ${ }^{4}$ Departamento de Métodos Cuantitativos en Economía y Gestión, Universidad de Las Palmas de Gran Canaria, Las Palmas Gran Canaria, Spain; ${ }^{5}$ Department of Public Health and Policy, University of Liverpool, Liverpool, UK

\subsection{6/jech-2021-SSMabstracts.38}

Background Fruit and vegetable $(\mathrm{F} \& \mathrm{~V})$ intake is an important modifiable risk factor for cardiovascular disease (CVD). Fiscal policies have been recommended to increase F\&V consumption, but their potential impact on health and inequalities are not well described.

We therefore explored the potential benefits of five different fiscal policies on F\&V consumption, CVD mortality and associated socio-economic inequalities in Madrid, Spain (total population $\sim 6,700,000$ residents).

Methods A modelling study using a comparative risk assessment approach.

We compared five policy options:

Option 1) reducing F\&V Value Added Tax (VAT) from 4\% to $0 \%$;

Option 2) a $\mathrm{F} \& \mathrm{~V}$ price discount of $10 \%$;

Option 3) a targeted F\&V subsidy of 50\% for low-income families;

Option 4) a mass media campaign promoting F\&V consumption, and

Option 5) The combination of Options 3 and 4.

We computed F\&V intake, CVD deaths prevented or postponed (DPPs), and CVD mortality inequalities between quintiles of area-level socio-economic status (SES) in 2021-2035, compared to a no-intervention scenario based on observed trends in F\&V intake and CVD mortality. We used estimates for price elasticities, pass-through of policies, and F\&V effect measures from relevant sources. We conducted sensitivity analyses through 5,000 Monte Carlo iterations with wide margins using the statistical software R.
Results A combined mass media campaign and 50\% targeted discount could prevent or postpone approximately $700 \mathrm{CVD}$ deaths (95\% UI: $50-1000)$ in 2021-2035, representing a $1 \%$ reduction in total CVD mortality. A $50 \%$ targeted discount alone might generate some 450 DPPs, (95\%UI: $300-$ 700), a $10 \%$ universal subsidy approximately 300 DPPs, (95\% UI: 100 - 450), a mass media campaign some 200 DPPs, (95\%UI: 150 - 400), and 0\% VAT 100 DPPs, (95\%UI: $50-$ 200). Every option would modestly reduce socio-economic inequalities in CVD mortality, with the combined policy achieving the greatest reduction, approximately $10 \mathrm{DPPs}$ / 100,000 population between highest- and lowest-SES areas.

Conclusion Fiscal policies and mass media campaigns targeting F\&V consumption applied singly, or ideally in combination, could reduce CVD mortality and decrease social inequalities in a large southern European urban population.

\section{OP39 ESTIMATING GEOGRAPHICAL INEQUALITIES IN FRUIT AND VEGETABLE INTAKE IN LIVERPOOL, UK: A SPATIAL MICROSIMULATION APPROACH}

${ }^{1}$ Ellen Schwaller*, ${ }^{2}$ Mark Green, ${ }^{1}$ Christodolous Kypridemos, ${ }^{3}$ Grace Patterson, ${ }^{1}$ Martin O'Flaherty. 'Public Health, Policy and Systems, University of Liverpool, Liverpool, UK; ${ }^{2}$ Geography and Planning, University of Liverpool, Liverpool, UK; ${ }^{3}$ Epidemiology and Population Health, University of Liverpool, Liverpool, UK

\subsection{6/jech-2021-SSMabstracts.39}

Background Individual-level data of health outcomes and their drivers are rarely available at a neighbourhood-level. While national-level surveys may provide insights at a regional scale, these data do not help us understand how complex problems such as poor diet affect communities within cities where policies can be leveraged for better health outcomes. Spatial microsimulation (SMS) can be used to approximate individuallevel data for small areas. The aim of our study is to estimate small area fruit and vegetable intake for Liverpool (UK) to explore geographical inequalities in diet.

Methods Spatial microsimulation leverages individual level data and fits it to distinct spatial data. The fitting is based on selected constraint variables contained within both data sets. We perform the SMS using individual-level data extracted from the National Dietary and Nutritional Survey (2014-2018, $n=2018$ ) and aggregated data from 2011 UK Census at Lower Super Output Area (LSOA). Our outcome variable is daily servings of fruits and vegetables consumed. Constraint variables include age, sex, highest level of qualifications, self-reported health, and national statistics-socioeconomic classification. Constraints were chosen based on literature of key determinants of dietary predictors existing across both data sets. An iterative proportional fitting approach is executed in $\mathrm{R}$ to estimate fruit and vegetable intake for each LSOA. We use internal validation via model fit statistics and external validation through comparing estimates to an Eating Habits Survey $(n=1724)$ with partial representation of Liverpool.

Results We estimated that $25 \%$ of adults aged 16 and up in Liverpool meet their daily recommendation of fruit and vegetable intake (5 or more servings), with $7 \%$ consuming fewer than 1 serving a day. Fruit and vegetable consumption was unevenly distributed across the city, with consumption of 5 or more servings ranging from $18 \%$ to $37 \%$. There were higher levels of fruit and vegetable consumption among populations in the least deprived neighbourhoods with $35 \%$ meeting daily 
recommendations (versus 22\% in most deprived). Preliminary model validation appears to suggest estimates are suitable for use.

Conclusion Our study presents a novel framework for estimating fruit and vegetable consumption for small areas and within cities. We find low levels of fruit and vegetable consumption in Liverpool, with wide geographical inequalities in consumption by level of deprivation. These results were largely supported by internal/external validation. Producing small area statistics can support better nuanced decision-making including geographical targeting of interventions, especially for local authorities, as well as provide robust inputs for other modelling methods (e.g. agent-based modelling).

\section{OP40 'THERE IS NO SILVER BULLET' HOW PARLIAMENTARY DEBATE ON THE UK SOFT DRINKS INDUSTRY LEVY CHANGED OVER TIME (2014-2020): AN APPLIED THEMATIC ANALYSIS}

${ }^{1}$ Catrin Penn-Jones*, ${ }^{1}$ Emma Lawlor, ${ }^{1}$ Hannah Forde, ${ }^{2}$ Tarra Penney, ${ }^{3}$ Steven Cummins, ${ }^{1}$ Martin White. 'Centre for Diet and Activity Research, MRC Epidemiology Unit, University of Cambridge, Cambridge, UK; ${ }^{2}$ School of Global Health, York University, Toronto, Canada; ${ }^{3}$ Department of Public Health, Environments and Society, London School of Hygiene and Tropical Medicine, London, UK

\subsection{6/jech-2021-SSMabstracts.40}

Background Announced in March 2016 and implemented in April 2018, the UK Soft Drinks Industry Levy (SDIL) aims to incentivise the reformulation of soft drinks. The SDIL has successfully decreased the amount of sugar in UK soft drinks, and purchased in soft drinks. Consequently, the SDIL has been widely applauded as a policy success. SSB taxation in other countries, however, has not been as successful and in some cases the policy was retracted. We aimed to develop an understanding of why the SDIL was deemed successful by analysing parliamentary debate two years before its announcement until two years post-implementation (2014-2020).

Methods Searches of Hansard were conducted for parliamentary debate transcripts discussing the SDIL across three key time periods: Pre-Announcement (01/01/14-15/03/16), Announcement - Implementation (16/03/16-5/04/18), and Post-Implementation (6/04/18-16/03/20). 218 transcripts containing eligible search terms were identified, and 179 included in the analysis after screening for relevance. Applied thematic analysis was conducted in 5 stages: familiarisation and creation of initial codebooks, independent second coding, codebook finalisation through team consensus, final coding of the dataset to the complete codebook and theme finalisation through team consensus.

Results Common issues raised in discussions across the timeline were: the ring-fencing of SDIL funds for pro-social causes, the scale of the health problems associated with excess sugar consumption, and the role of celebrities in making the SDIL a high profile issue. MPs acknowledged that the SDIL is not a 'silver bullet' for obesity prevention: it was positioned as either a 'good start' with other interventions required or that other interventions were needed instead. Distinct themes were that, due to early reformulation, the SDIL was discussed as 'having worked' one year prior to implementation. This notion persisted until the end of the timeline. These themes will be further refined to identify temporal patterns and trends. Additional results will be submitted before the June 28th deadline.
Population Health Relevance The SDIL is unique: it is a flagship policy for the UK Childhood Obesity Strategy and has cross-party support. Understanding why and how the SDIL made it onto the statutes, and why it was politically successful is vital for using policy instruments to improve population health in future. This work will aid understanding of the parliamentary process surrounding the SDIL, and could provide insight for those wishing to extend the SDIL to other products or implement SSB taxes elsewhere.

\section{OP41 EVALUATION OF A NATURAL EXPERIMENT TO INCREASE AVAILABILITY OF HEALTHIER SNACK FOODS IN VENDING MACHINES USING INTERRUPTED TIME SERIES ANALYSIS}

Charlotte Evans*. Food Science and Nutrition, University of Leeds, Leeds, UK

\subsection{6/jech-2021-SSMabstracts.41}

Background The food environment plays a key role in access and availability of healthier food and drink choices. Community based vending machines are one source of energy dense snack foods high in saturated fats, sugars and salts, particularly for young people. The aim of this research was to evaluate the increased availability of healthier options of different types of snack products sold in vending machines situated in English leisure centres. The natural experiment was managed by Leeds city council with vending provision by Wilkes vending company and support from Public Health England (PHE).

Methods The intervention sought to increase the availability of healthier food items within 18 vending machines in Leeds leisure centres over three iterative phases during 39 weeks between September 2018 and May 2019. Products were altered to meet agreed nutrition criteria at each phase based on the Government Buying Standards for Food and Catering Services (GBSF) and taking into consideration calorie guidelines within PHE's sugar reduction programme, and product availability. A quasi experimental trial design was used with interrupted time series analysis and segmented regression techniques adjusting for underlying trends, correlation between data points and holiday weeks when footfall is generally lower. The primary outcomes were the changes in level and trend of weekly purchased energy in phase 1 and phase 2. Secondary outcomes included phase changes in levels and trends for saturated fats, sugars, salt and purchased units.

Results Total energy, saturated fats, sugars and salt from products purchased all significantly reduced in phase 1 compared with baseline and although did not reduce further at phase 2 when more stringent standards were implemented, nutrients purchased continued to be lower compared with baseline. Trend changes during phase 1 were non-significant for all nutrients; however, during phase 2 there were upward trends for energy, saturated fats and salt. The total weekly amount of energy purchased by all machines reduced by $244,256 \mathrm{kcal}(95 \%$ CI $-399,456$ to $-89,057$ $\mathrm{kcal}$ ), between baseline and phase 1 . The reductions for saturated fats, sugars and salt were 4,917g (95\%CI -8884 to $-950 \mathrm{~g}), 23,868 \mathrm{~g}(95 \% \mathrm{CI}-38,369$ to $-9,366 \mathrm{~g})$ and $425 \mathrm{~g}$ (95\%CI -644 to $-207 \mathrm{~g}$ ) respectively. Weekly sales varied over time and were lower in phase 1 but recovered in phase 2 . 